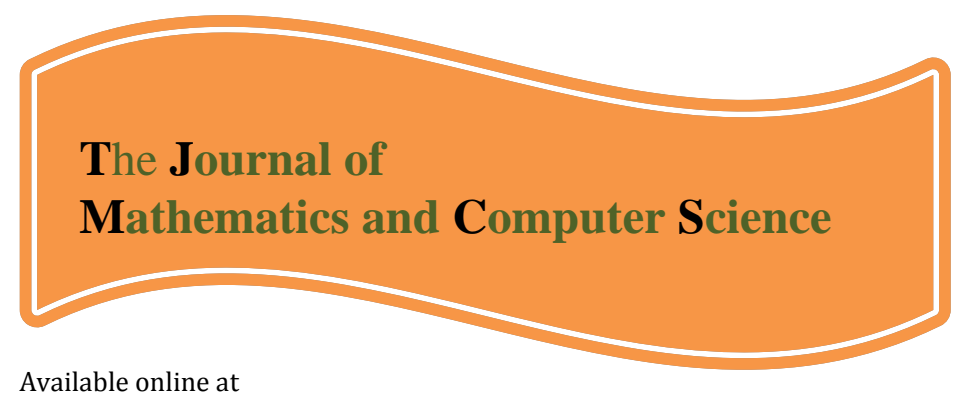

http://www.TJMCS.com

The Journal of Mathematics and Computer Science Vol .2 No.2 (2011) 337-347

\title{
Fuzzy Model Identification for Intelligent Control of a Vehicle Speed Limit
}

\author{
Jafar Pouramini', Ahmad Saeedi ${ }^{2}$ \\ Payame Noor University, pouramini@yahoo.com \\ Mofid University, asaaeedi@yahoo.com
}

Received: August 2010, Revised: November 2010

Online Publication: January 2011

\begin{abstract}
The need to increase road safety is a major concern, with millions of road users and pedestrians being killed in traffic accidents each year. Static speed limit signs are conventionally used to assist motorists in safe selection of speeds. Although appropriate to use under near ideal conditions, such signs fail to provide accurate information on speed selection when traffic and environmental conditions are less than ideal. To develop a variable speed limit system that utilizes fuzzy control technology to identify speed limits appropriate for differing environmental conditions the problem of structure identification of a fuzzy model was studied. This paper presents a fuzzy rulebase combined controller, which is a fuzzy rule-based combination of linear controllers, for nonlinear systems subject to parameter uncertainties. The paper discusses potential benefits and limitations associated with the model. The main interest has been on building fuzzy relationship models that are expressed by set of fuzzy linguistic propositions derived from the experience of the specialists. The proposed model was tested on a set of experimental data and against specialist knowledge the comparison was satisfactory regarding the aims of the model. This system dynamically updates posted speed limits to better reflect prevailing traffic and environmental conditions.
\end{abstract}

Keywords: Variable Speed Limit (VSL)-Intelligent Transport Systems (ITS)Traffic Control Devices - Fuzzy Logic

\footnotetext{
${ }^{1}$ Jafar pouramini: Payame Noor University, Computer Engineering Faculty, Software Engineering.

${ }^{2}$ Ahmad Saeedi: Mofid University, Economics Group, Applied Mathematics.
} 


\section{INTRODUCTION}

Traffic signs on roads to secure and sustain lives of citizens have played an important role. More these signs are accurately its effect in reducing road accidents is higher. But if the information of these signs is not sufficiently accurate loose its effect. Because if signs have not enough accuracy to provide information to drivers cause confusion over drivers and so little attention to the signs.

One type of the traffic signs is signs indicate the speed limit. Passing the speed limit always is one of the reasons of death in traffic accidents. But these signs are not accurate enough because always and in any conditions show a fix number and do not react to changes around its circumferences. For example, the speed limit on a usual day with the speed limit in a snowy winter day is not equal. In this research the impact of using variable speed limit to reduce accidents has been verified and even in some cases, variable speed limits to control traffic volume has been used [1]-[3]. For example, when heavy traffic is generated, speed limit is reduced. As a result, the number of vehicles input to the critical traffic point is reduced and traffic staffs have more opportunity to unlock the traffic [4]-[6]. Sometimes drivers can not estimate the speed limit. Because of this many drivers have accident.

Fuzzy logic is a problem-solving technique that derives its power from its ability to draw conclusions and generate responses based on unclear, incomplete, ambiguous, and imprecise information. To simulate this process of human reasoning, it applies the mathematical theory of fuzzy sets. Fuzzy inference is the process of formulating a mapping from a known input value to an output value using fuzzy logic. The mapping then provides a basis from which decisions can be made, or patterns discerned. It has been proven that the system can effectively express highly non-linear functional relationships [7]. Fuzzy inference systems (FIS) have been successfully applied in fields such as data classification, automatic control, expert systems, and decision analysis and computer vision.

Fuzzy logic provides a practicable way to understand and manually influence the mapping behavior. Commonly, fuzzy logic uses simple rules to describe the system of interest, rather than analytical equations, making it easy to implement. An FIS contains three main stages, the fuzzification stage, the rule base and the defuzzification stage. The fuzzification stage is used to transform the so-called crisp values of the input variables into fuzzy membership values. Then, these membership values are processed within the rule-base, using conditional 'if-then' statements. The outputs of the rules are summed and defuzzified into a crisp analogue output value. The effects of variations in the parameters of a FIS can be readily understood and this facilitates calibration of the model. Following the evaluation of the rules, the defuzzification transforms the fuzzy membership values into a crisp output value. The complexity of a fuzzy logic system with a fixed input-output structure is determined.

Fuzzy logic modeling techniques could be classified into two categories, namely linguistic and Takagi-Sugeno-Kang (TSK). In linguistic models, such as Mamdani type, both the antecedent and consequence are fuzzy sets. While in the TSK model, the antecedent consists of fuzzy sets but the consequence is made up of linear equations. On the other hand, fuzzy rule base systems are divided into direct and indirect approaches. In direct approach to fuzzy modeling, the rules are extracted with interview and 
negotiations with expert(s). In the indirect approach to fuzzy modeling, implementing a fuzzy cluster analysis approach generates the rules. This approach is appropriate when historical data sets are available. In this paper direct approach was used because historical data are not available.

Application of Fuzzy Logic to Intelligent Transportation Systems is considered several times[9]-[12]. Speed control is the one of the important issue in Intelligent Transportation Systems. In this paper a model for speed control is presented according to vehicle and road conditions simultaneously.

\section{Architecture}

Speed limit on the road depends on various parameters. The most important parameters affecting speed limit according to experts is as follows: Horizontal visibility, temperature, road gradient, road traffic, one-way or two-way road, road width, road surface quality, vehicle type, humidity road surface, Quality vehicle tires, vehicle weight, distance from the front vehicle, bolt road

These parameters can be divided into two classes:

Vehicle-related parameters: vehicle type, quality vehicle tires, vehicle weight, distance from the vehicle front

Road-related parameters: horizontal visibility, road traffic, road width, road surface quality, humidity, road gradient, one-way or two-way road, bolt road.

The simplified schematic picture of approach is shown in Figure 1. This approach has two main components: Fuzzy Variable Speed Limit Sign System (FVSLS) and Fuzzy Vehicle Speed System (FVS). Speed limit for the ideal vehicle is computed by the FVSLS called $\alpha$. This is computed according to road-related parameters and transmitted to the passing vehicle (Figure 2). FVS controls vehicle speed by computing speed limit for the vehicle according to vehicle-related parameters and $\alpha$.

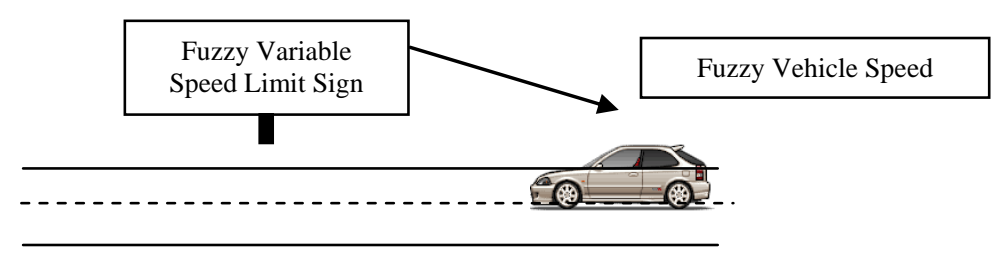

Figure 1. The simplified schematic picture of approach

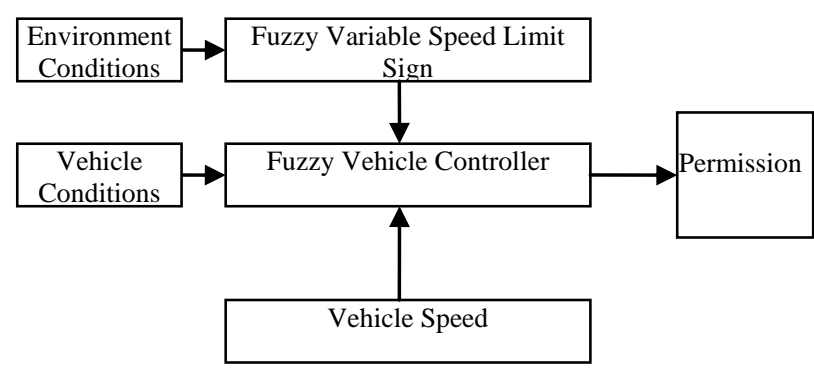

Figure 2. Block diagram of system

\section{Fuzzy Variable Speed Limit Sign System}

Road-related parameters divided into two classes: 
Road constant parameters: Parameters those are constant in a long time such as road quality, road width, one-way or two-way road, road gradient, bolt road.

Road variable parameters: Parameters that change at the moment such as road traffic, temperature, radius of visibility, humidity, road surface.

FVSLS should be installed at a specific location of road that shows speed limit for that location. Influence of road constant parameters in the speed limit is constant over a long time. So these parameters do not influence the speed limit at the moment. Speed limit in a range of road in the best road variable parameters called $\beta$.

Road variable parameters affect on speed at the moment.

$\mu$ : Effect of road variable parameters in speed limit. $\mu$ is calculated using fuzzy system.

$$
0<=\mu<=1
$$

\subsection{Fuzzy logic model}

In this section, designing a fuzzy system to compute variable $\mu$ is considered. Road parameters including temperature, humidity, horizontal visibility and traffic are measured by sensors that are fuzzy system inputs. In this system, the following linguistic variables are used:

Temperature: (freezing, normal) ranges from -30 to 70 Celsius.

Horizontal visibility: (low, medium, large) between 0 to 5000 meters.

Road surface humidity: (low, medium, large) between $0 \%$ to $100 \%$.

Traffic: (light, medium and heavy) between 0 to 1

Speed limit is computed by the equation

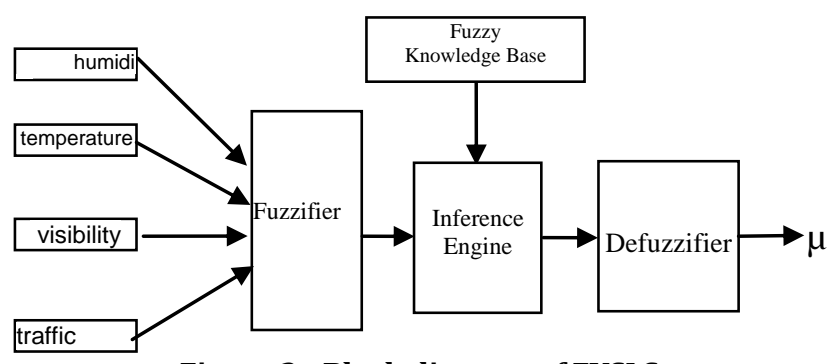

Figure 3 - Block diagram of FVSLS

The system output is between zero and one and is called $\mu$. Speed limit is obtained from

$$
\alpha=\mu * \beta
$$

Calculated speed limit $(\alpha)$ is transmitted to the passing cars. The schematic diagram (Figure 3) shows the FVSLS. The initial membership functions and rules are designed by experts' knowledge about the target system to be exploited. It refines the fuzzy 'if-then' rules and membership functions to describe the input/output behavior of a system. Graph of the membership functions for the fuzzy temperature variable and fuzzy humidity variable is shown in Figure 4, 5 respectively. 


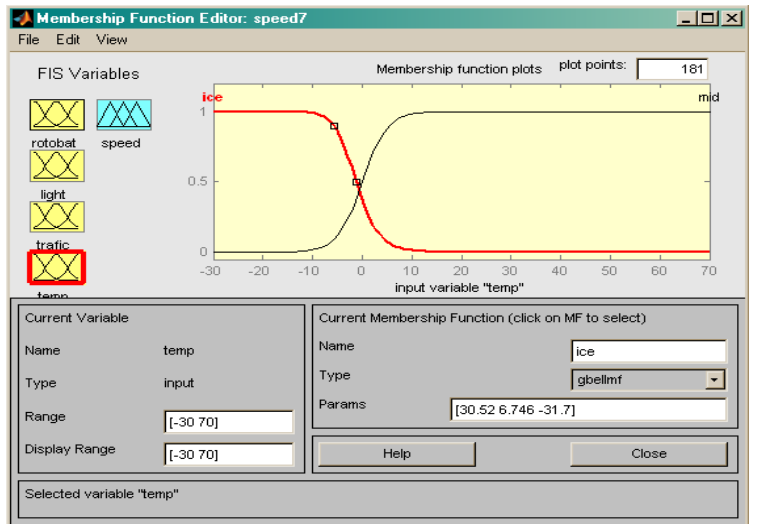

Figure 4. Membership functions for the fuzzy temperature variable

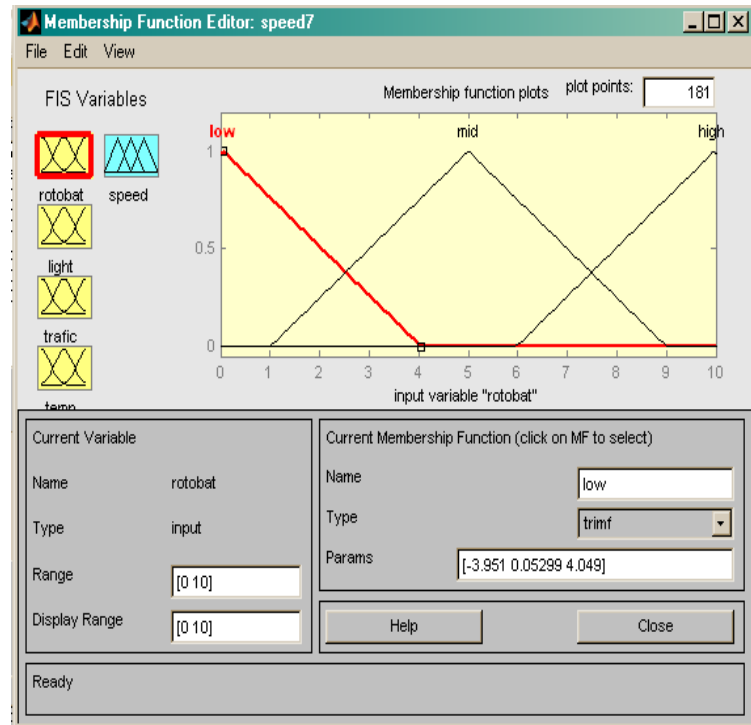

Figure 5. Membership functions for the fuzzy humidity variable

The AND and OR operators of Boolean logic exist in fuzzy logic, usually defined as the minimum and maximum, when they are defined this way, they are called the Zadeh operators. So for the fuzzy variables $\mathrm{x}$ and $\mathrm{y}[8]$ :

$\operatorname{truth}(\mathrm{X}$ and $\mathrm{Y})=\operatorname{Min}(\operatorname{truth}(\mathrm{X}) \operatorname{truth}(\mathrm{Y}))$

$\operatorname{truth}(\mathrm{X}$ or $\mathrm{Y})=\operatorname{Max}(\operatorname{truth}(\mathrm{X}), \operatorname{truth}(\mathrm{Y}))$

Various defuzzification methods were tested, the results were compared, finally average centroid method was selected because other defuzzification methods (e.g. largest of maximum defuzzification method), makes a sudden change in output that is not acceptable of specialists.

$$
\mathrm{y}^{*}=\left(\sum^{\left.\mathrm{M}_{\mathrm{l}=1} \mathrm{y}^{-\mathrm{l}} \mathrm{w}_{\mathrm{l}}\right) /\left(\sum^{\mathrm{M}_{\mathrm{l}}=1} \mathrm{w}_{\mathrm{l}}\right)}\right.
$$

Considering the number of linguistic variables and interviews with experts was producing 54 rules (Fig. 6, 7). FVSLS has been simulated in Simulink (Fig. 8). 


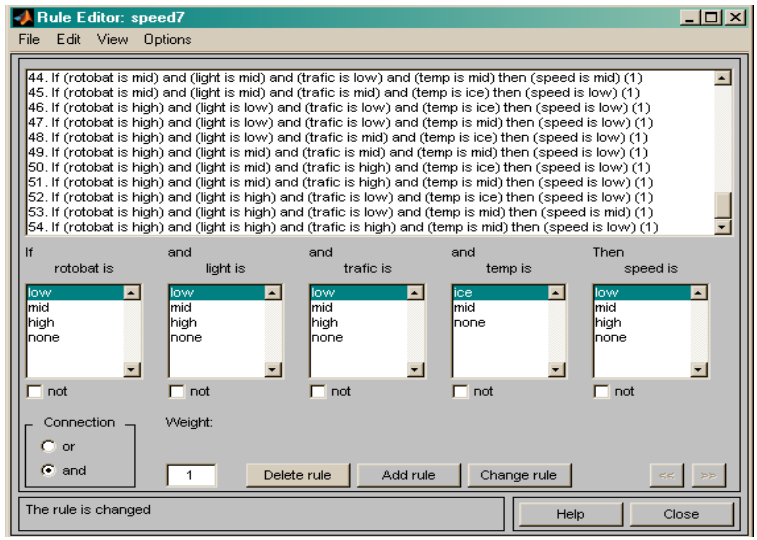

Figure 6. 54 Fuzzy rules for 4 inputs

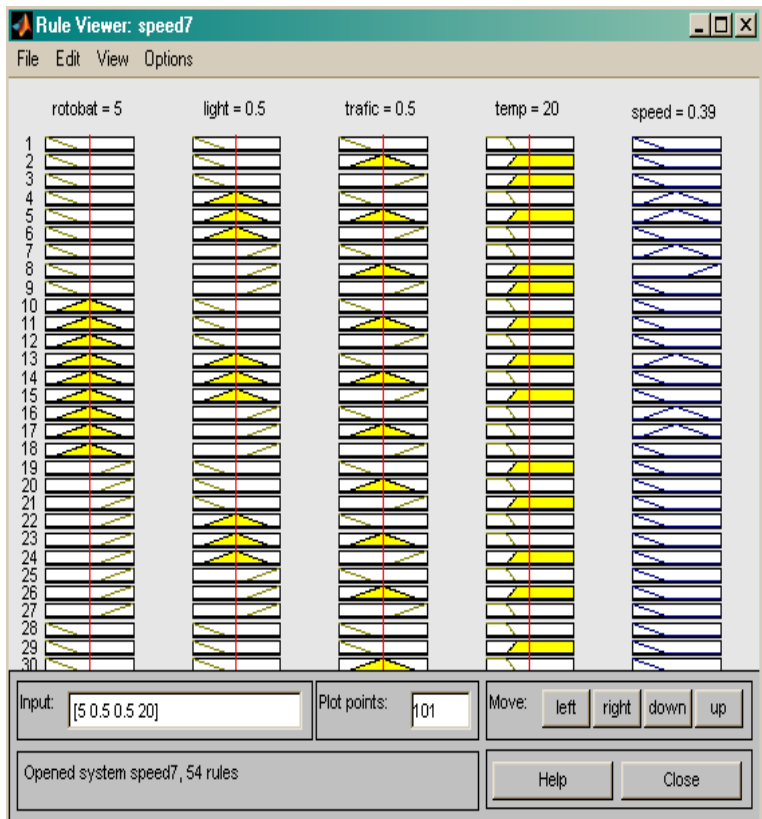

Figure 7. Rule viewer for 4 inputs and one output

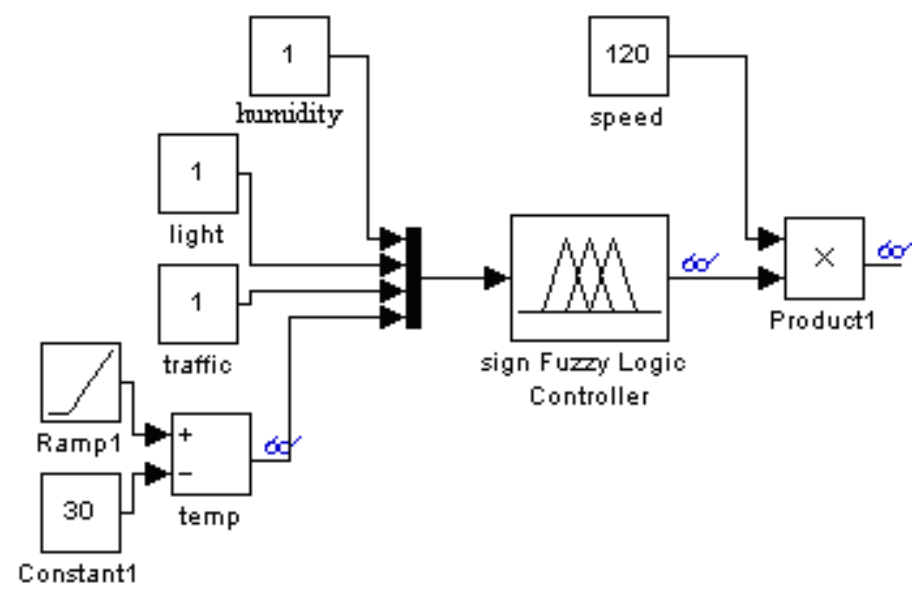

Figure 8: Simulink simulation of FVSLS

Simulations performed under the specified conditions (i.e. speed $120 \mathrm{~km} / \mathrm{h}$, humidity $1 \mathrm{~mm}$, traffic $1 \mathrm{v} / \mathrm{m}$, light 1 ). The result obtained by Simulink in MATLAB is shown in Figure 9 . It can be 
seen from Figure 9, 10 that speed limit how is dependent on variation in temperature from $20^{\circ} \mathrm{C}$ to $70^{\circ} \mathrm{C}$.

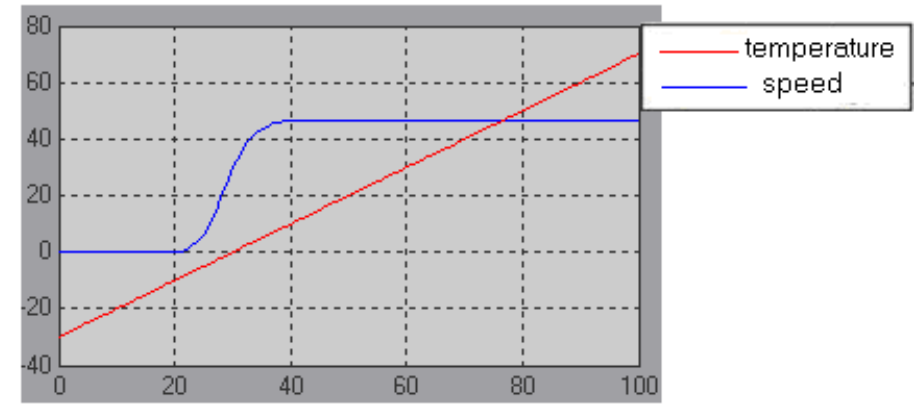

(a)

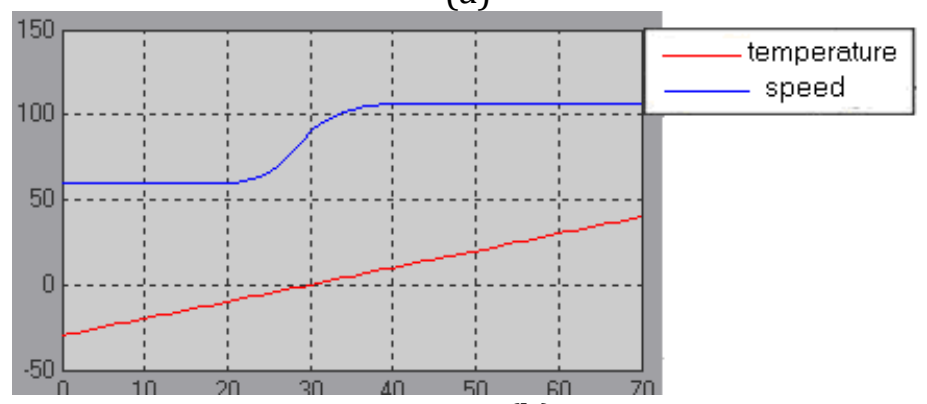

(b)

Figure 9. Dependence of speed limit on temperature (a) humidity $1 \mathrm{~mm}$ (b) humidity $0.5 \mathrm{~mm}$

\section{Fuzzy Vehicle Speed System}

In this section designing the second component of the system (FVS) (sec. 3) is considered. FVS is a Mamdani fuzzy system. Schematic FVS is shown in Figure 10. It has three parameters as input:

Tire condition (good, average, poor): this parameter in terms of tire performance status is determined. Status rubber also based on distance traveled by the tire is measured. This article assumes that replacement tires are all together.

Distance from the front vehicle (low, medium, high): This parameter according to meters and shows the distance from the vehicle in front of the car is the same line.

Vehicle weight (low, medium, high): Vehicle weight to be considered as base and added loads (Weight of passengers and cargo) add to the vehicle weight. For this purpose 27 rules are produced (Figure 11, 12).

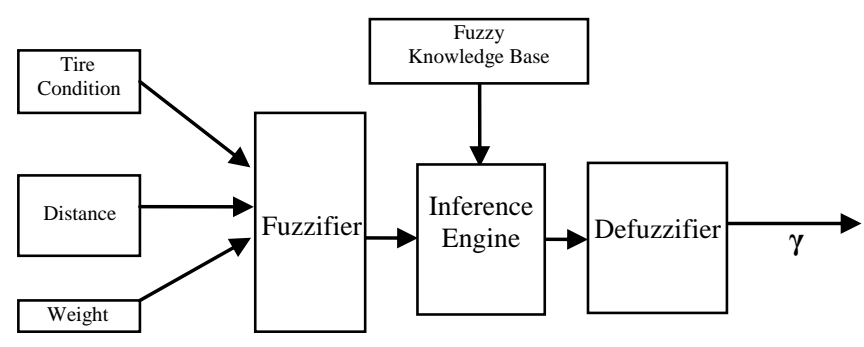

Figure 10. Block diagram of the FVS 


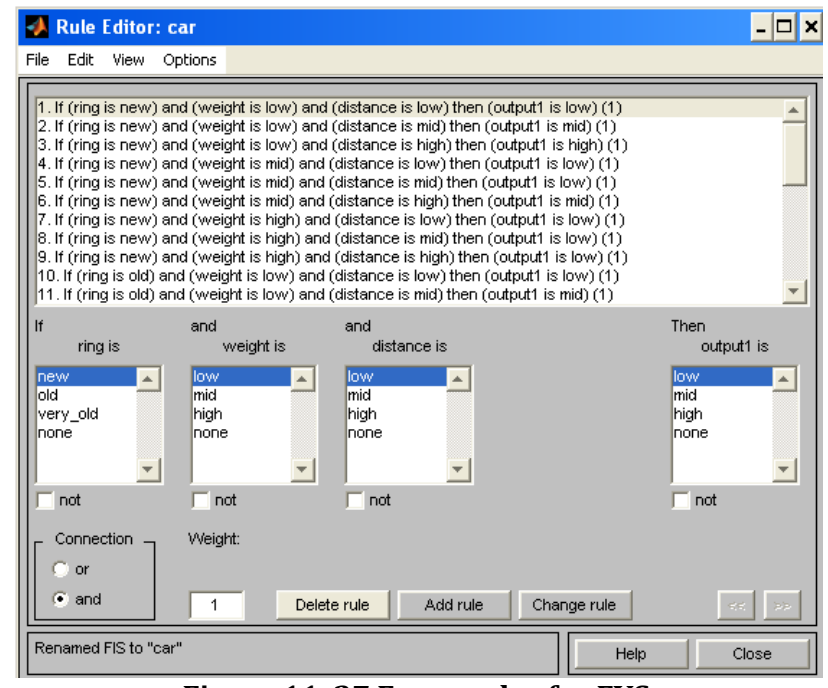

Figure 11. 27 Fuzzy rules for FVS

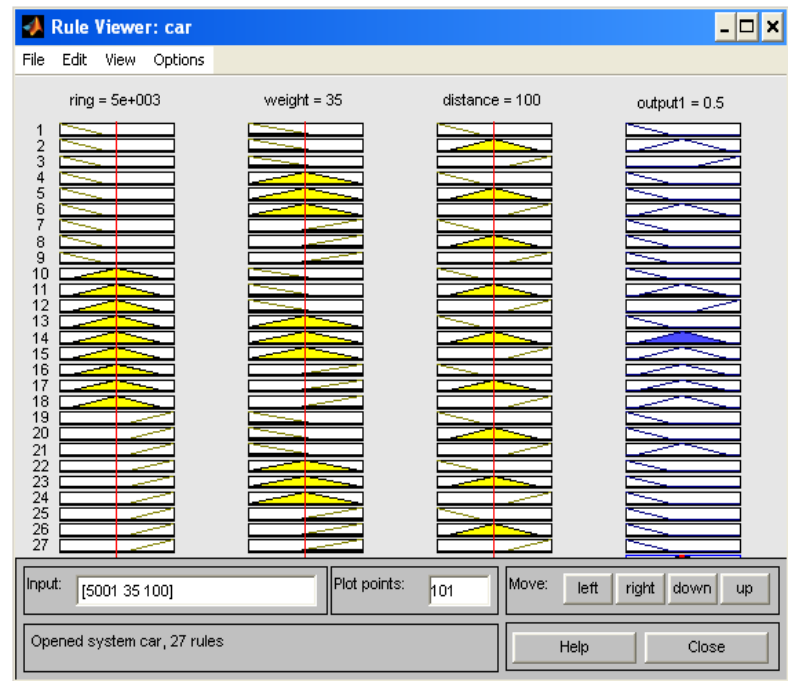

Figure 12. Rule viewer for FVS

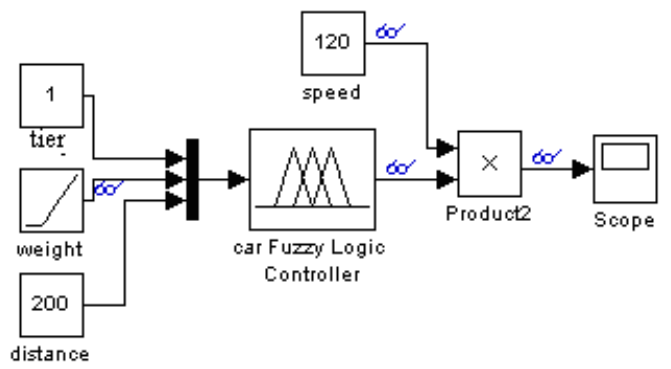

Figure 13. Simulink Simulation of FVS

FVS output is between zero and one and is called $\gamma$. Speed limit of vehicle is obtained from

$$
\lambda=\gamma^{*} \alpha
$$

(6)

FVS has been simulated in Simulink (Fig. 13).Simulations performed under the specified conditions (i.e. Distance $200 \mathrm{~m}$, Tire 1 day). The results obtained by Simulink in MATLAB are shown in Figure 15. It can be seen from Figure 14 that speed limit how is dependent on variation in weight 0-70 ton. 


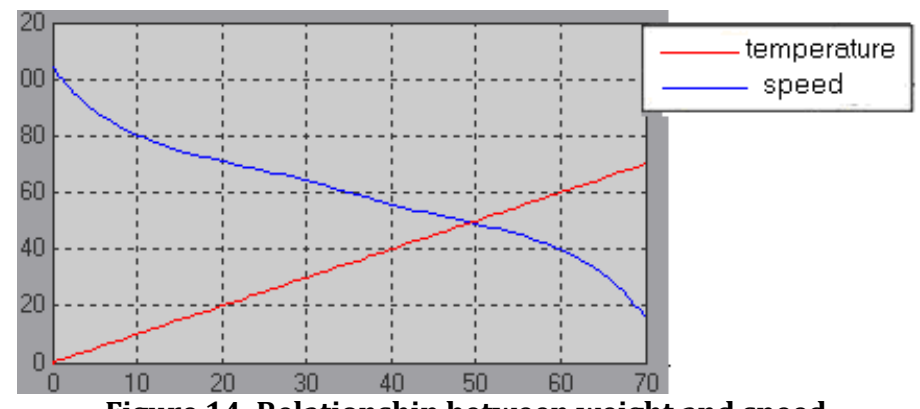

Figure 14. Relationship between weight and speed

"And" and "or" operators, respectively, defined (3), (4). Various defuzzification methods were tested, the results were compared, finally average centroid method was selected because other defuzzification methods (e.g. largest of maximum defuzzification method), makes a sudden change in output that is not acceptable of specialists (3) [7].

\section{Test and Validation}

The model components are linked up in MATLAB using the Simulink interface so that simulation results can be seen and the component parameter can be simply changed in order to experiment on different values. Figure 15 shows Simulink Simulation of the model. Obtained outputs were compared with experts. With the studies and according to experts, 87 percent of the speed provided by the system was approved by experts.

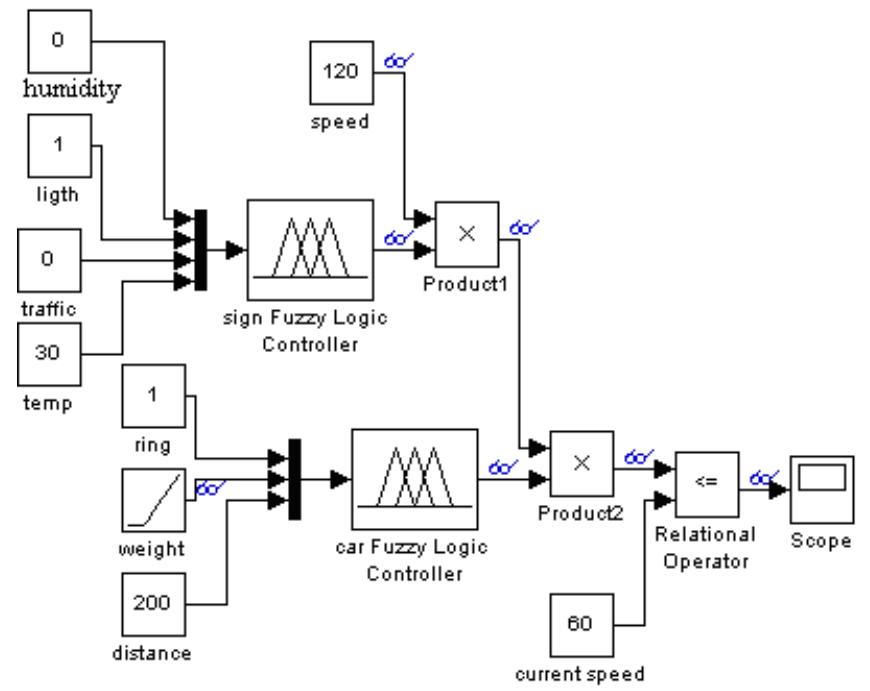

Figure 15. Simulink simulation of the model

As an example, Fig. 17 shows a selected plot. Vehicle speed is $60 \mathrm{~km} / \mathrm{h}$. Fig. 16 shows the increase in vehicle weight, speed limit is reduced to where the speed limit is less than $60 \mathrm{~km} / \mathrm{h}$. In these circumstances the model replies and alarms. If the vehicle speed exceeds from $60 \mathrm{~km} / \mathrm{h}$ then model alarm and prevent increasing speed. 


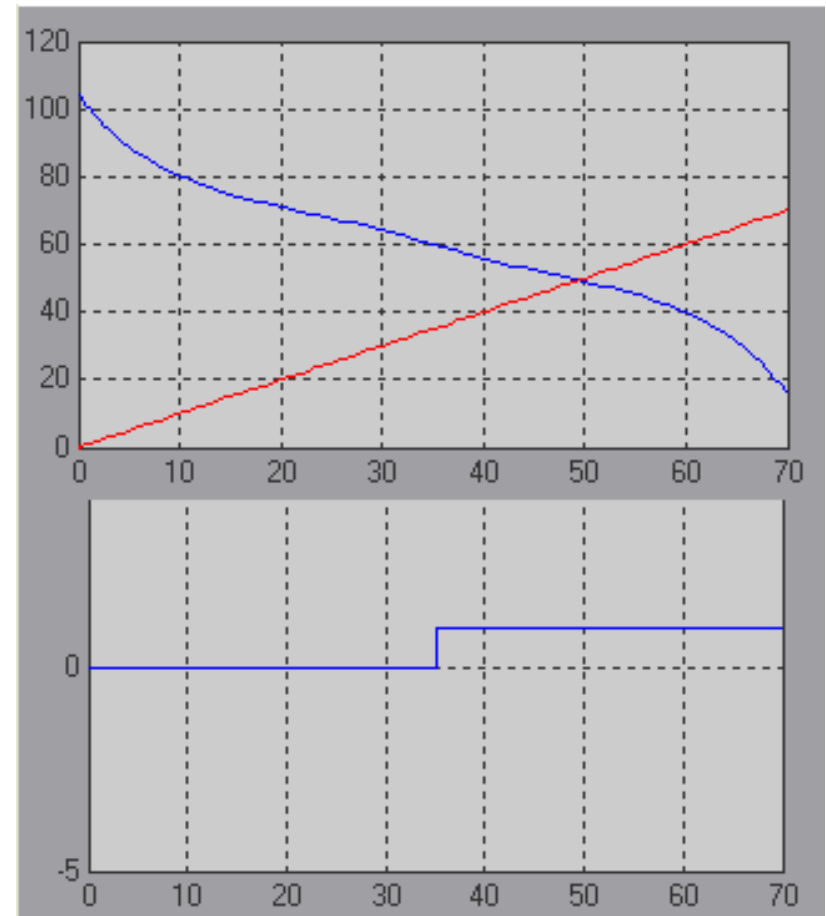

Figure 16. Typical the model response conclusions

\section{Conclusion}

In this investigation we considered the problem of identifying a fuzzy Model for Intelligent Control of a Vehicle Speed Limit. This model is a fuzzy rule-based model to control speed of vehicle by considering road and vehicle conditions.

The main interest has been on building fuzzy relationship models that are expressed by set of fuzzy linguistic propositions derived from the experience of experts. The proposed model was tested on a set of experimental data. The empirical results with real data set indicate that the model computes speed limit and controls vehicle speed more effective than conventional method. Therefore, it can be used as an appropriate model for intelligent control of a Vehicle Speed Limit. Further work underway includes design a nero-fuzzy system to compute speed limit.

\section{References}

[1] Proceedings of the 11th Annual Meeting of ITS America, 2001 I Variable Speed Control: Technologies and PracticeVirginia P. Sisiopiku, Ph.D.Assistant Professor, Michigan State University,3546 Engineering Building, E. Lansing,

[2] Research Report No. UVACTS-13-0-43 July, 2004 Development and testing of variable speed limit control logics for works zones using simulation by: Sivanaga S. Yadlapati Dr. Byungkyu "Brian" Park

[3] The potential for variable speed control to improve safety on urban freeways ,Submitted for the Transportation Association of Canada Annual Conference to be held October 14-17, 2007 in Saskatoon, Saskatchewan Submitted for the Session entitled: Travel Speed Management Bruce Hellinga Associate Professor, Dept. of Civil Engineering University of Waterloo, ON N2L 3G1

[4] Maintenance Management 2009 Presentations from the 12th AASHTO-TRB Maintenance Management Conference July 19-23, 2009 Annapolis, Maryland Hosted by Maryland State Highway Administration Sponsored by Transportation Research Board American Association of State Highway and Transportation Officials Federal Highway Administration July 2009 Transportation Research Board 500 Fifth Street, NW Washington, DC 20001 www.TRB. 
[5] An Investigation On The Environmental Benefits Of a Variable Speed Control Strategy by Zhong Wang and C. Michael Walton Research Report SWUTC/06/473700-00072-1 Southwest Region University Transportation Center for Transportation Research University of Texas at Austin, TX 78712 September 2006

[6] Dynamics of Variable Speed Limit System Surrounding Bottleneck on German Autobahn Transportation Research Record: Journal of the Transportation Research Board 0361-1981 Volume 1978 / 2006 149-159 Wednesday, February 28, 2007

[7] Zimmermann ,H.J.[1991], Fuzzy Set Theory and Its Applications, Kluwer Acramic Publications, Boston.

[8] Zadeh, L.A[1962], “Fuzzy Set”, Informat. Control,8 ,pp. 338-353.

[9] X.Yang, M.Moallem, and R. V. Patal,. 2005 "Alayerd goal-oriented fuzzy motion planning strategy for mobile robot navigation” in IEEE Trans. Syst.,Man,Cybern. B, Cybern. vol. 35,no. 6, pp.1214-1224, Dec. 2005

[10] Lin, Q., Kwan, B.W., and Tung, L.J., (1997). Traffic Signal Control Using Fuzzy Logic, Proc. IEEE International Conference on Fuzzy Systems 1997, pp 1644-1649.

[11] Beauchamp-Baez,G., Rodriguez-Morales, E., and Muniz Marrero, E.,L., (1997). A Fuzzy Logic Based Phase Controller for Traffic Control, Proc. IEEE International Conference on Fuzzy Systems 1997, pp 1533-1539.

[12] Intelligent Speed Adaptation Using a Self-Organizing Neuro-Fuzzy Controller David Partouche Michel Pasquier Anne palanzani Proceedings of the 2007 IEEE Intelligent Vehicles Symposium Istanbul, Turkey, June 13-15, 2007 